УДК 622.276:552.578

МЕТОДИКА ОЦЕНКИ ОБЪЕМОВ СЖИГАЕМОГО В ФАКЕЛАХ

НЕУВЛАЖНЕННОГО МЕТАНА И ЕГО ЭМИССИОННЫХ

СОСТАВЛЯЮЩИХ

\title{
METHODOLOGY FOR ESTIMATING THE VOLUMES OF UNMOIDED METHANE BURNED IN TORCHES AND ITS EMISSION COMPONENTS
}

\author{
И.Х. Асадов \\ Национальное аэрокосмическое агентство, г. Баку, \\ Азербайджанская Республика \\ В.М. Рамазанов
}

Азербайджанский государственный аграрный университет, г. Баку, Азербайджанская Республика

Ilgar H. Asadov

National Aerospace Agency, Baku, Azerbaijan Republic

\section{Vagif M. Ramazanov}

Azerbaijan State Agrarian University, Baku, Azerbaijan Republic e-mail: asadzade@rambler.ru

Аннотация. В статье рассматривается разработанная авторами методика оценки объема неувлажненного метана, сжигаемого в факелах и его эмиссионных составляющих. Разработан наземно-бортовой - космической метод для определения суммарного сухого объема сжигаемого в факелах метана, а также для определения эмиссионных компонентов факелов углеводородного природного газа, приводящих к нагреву атмосферы. Получена 
формула для определения верности результатов спутниковых, бортовых (БПЛА) и модельных измерений. Доказательство верности результатов измерений позволяет определить общий объем сжигаемого газа в факелах. Coставлен алгоритм реализации предложенного метода, включающего известный метод прокси $\mathrm{CH}_{4}$ измерений.

Abstract. The article discusses the methodology developed by the authors for assessing the volume of unmoistened methane burned in flares and its emission components. A ground-based - space-based method has been developed to determine the total dry volume of methane burnt in flares, as well as to determine the emission components of hydrocarbon natural gas flares, leading to heating of the atmosphere. A formula for determining the accuracy of the results of satellite, airborne (UAV) and model measurements has been obtained. An algorithm for the implementation of the proposed method, including the well-known method of proxy $\mathrm{CH}_{4}$ measurements, is compiled.

Ключевые слова: прокси измерения; парниковый эффект; сжигание метана; измерения; рассеяние света

Key words: proxy measurements; Greenhouse effect; combustion of methane; measurements; light scattering

\section{Введение}

Обычно сжигаемые в факелах газы имеют разный состав [1]. Состав сжигаемого газа, в первую очередь, зависит от источника этого газа. Например, природный газ более чем на 90 \% состоит из метана. Газы, сжигаемые на нефтеперерабатывающем производстве, обычно содержат смесь углеводородов. Однако газы, поступающие из захороненных мусорных полигонов, содержит смесь метана и двуокиси углерода. При этом газы, сжигаемые в факелах, всегда увлажнены и содержат некоторое количество влаги. Влага, 
содержащаяся в сжигаемом газе, испаряясь, увеличивает влажность нижних слоев тропосферы, что приводит к появлению дополнительной погрешности при определении объемов сухого метана и двуокиси углерода.

Сжигание природного газа является важным фактором увеличения содержания парниковых газов в глобальном масштабе. Согласно Парижскому соглашению по климату, такое увеличение не должно превышает 2 \% того прироста, который предусмотрен по отдельным странам [2]. Согласно [3], эмиссии парниковых газов, таких как $\mathrm{CO}_{2}, \mathrm{CH}_{4}, \mathrm{~N}_{2} \mathrm{O}$ главным образом появляются при сжигании ископаемых типов топлива (нефть, газ, уголь). Сжигание природного газа практикуется в нефтегазовой отрасли промышленности для уничтожения нежелательных воспламеняющихся газовых компонентов. Согласно [4-7], наиболее существенными, неконденсируемыми парниковыми газами являются $\mathrm{CO}_{2}$ и $\mathrm{CH}_{4}$, которые, поглощая уходящее инфракрасное излучение Земли, приводят к общему потеплению климата. Следовательно, важно иметь достаточно достоверные методы для точной оценки как таких эмиссий, возникающих при антропогенной деятельности, так и всего сухого объема метана, сжигаемого в факелах.

Согласно [4], данные, получаемые методами наземных измерений содержания $\mathrm{CO}_{2}$ и $\mathrm{CH}_{4}$ в атмосфере, были недостаточны для принятия решений по всему континентальному масштабу. Как результат, в 2009 г. был запущен спутник GOSAT, предназначенный для проведения достаточно точных измерений $\mathrm{CO}_{2}$ и $\mathrm{CH}_{4}$ в нижней тропосфере в коротковолновом инфракрасном диапазоне (SWIR). В результате функционирования этого спутника появилась возможность определить региональные значения содержания $\mathrm{CO}_{2}$ и $\mathrm{CH}_{4}$, используя данные GOSAT (колонные мольные фракции $\mathrm{CO}_{2}$ в сухом воздухе $\left(\mathrm{XCO}_{2}\right)$ и $\left.\mathrm{CH}_{4}\left(\mathrm{XCH}_{4}\right)\right)$, двумя методами:

- прокси метод;

- метод использования прямых измерительных величин физических показателей. 
В настоящей статье анализируется влияние влажности атмосферы на оценку указанных парниковых эмиссионных составляющих факелов сжигания $\mathrm{CH}_{4}$ в атмосфере. На этой основе разработана методика вычисления общего сухого объема сжигаемого метана в факелах.

\section{Краткое изложение прокси метода}

Согласно [1], для измерения концентрации $\mathrm{CH}_{4}$ на длине волны 1.65 мкм со спутника (SCIAMACHY, GOSAT) используются вышеуказанные два метода, разница между которыми заключается в том, что в методе физических измерений учитываются свойства рассеяния как поверхности земли, так и атмосферы.

$\mathrm{B}$ методе прокси $\mathrm{CO}_{2}$ измерений дополнительно проводятся измерения $\mathrm{CO}_{2}$ на длине волны 1.6 мкм, что позволяет компенсировать взаимное влияние атмосферного рассеяния на результат измерения как $\mathrm{CH}_{4}$, так и $\mathrm{CO}_{2}$. Изза близости длин волн измерений (1.6 мкм $\left(\mathrm{CH}_{4}\right)$ и 1.6 мкм $\left.\left(\mathrm{CO}_{2}\right)\right)$ эффектны атмосферного рассеяния оказываются почти одинаковыми, что позволяет определить колонную мольную фракцию метана по формуле:

$$
X_{\mathrm{CH}_{4}}=\left(\frac{\Omega_{\mathrm{CH}_{4}}}{\Omega_{\mathrm{CO}_{2}}}\right) \cdot X_{\mathrm{CO}_{2}}
$$

где $\mathrm{X}_{\mathrm{CO} 2}$ - сухое мольное соотношение $\mathrm{CO}_{2}$ в колонной смеси, величина которой извлекается из модели «Carbon Tracker» $[1,4]$;

$\Omega_{C H 4}-$ результат измерения $\mathrm{CH}_{4}$ на длине волн 1.65 мкм;

$\Omega_{\mathrm{CO} 2}$ - результат измерения $\mathrm{CO}_{2}$ длине волны 1.61 мкм.

На рисунке 1 показаны спектральные линии поглощения $\mathrm{CO}_{2}$ на спектральном диапазоне 1.59-1.62 мкм [1]. 


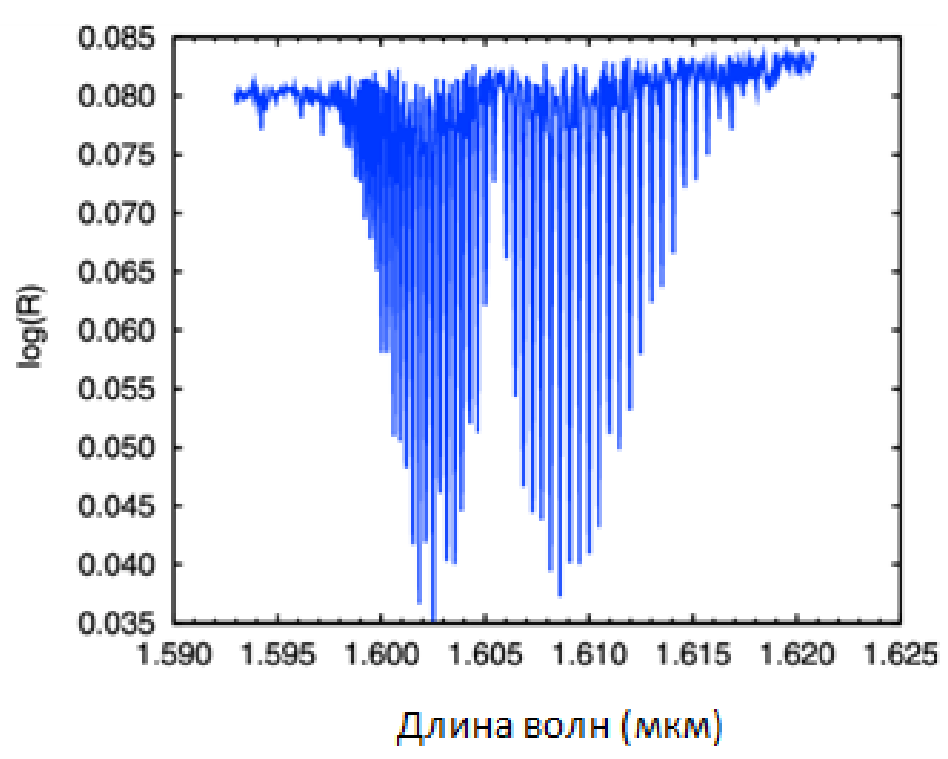

Рисунок 1. Спектральные линии поглощения $\mathrm{CO}_{2}$ в интервале длин волн $1.59-1.62$ мкм [1]

\section{Предлагаемый метод}

Для определения парниковых эмиссионных продуктов сжигания попутного газа и влияния атмосферного аэрозоля на указанную взаимосвязь и дальнейшего определения сухого объема сжигаемого метана в факелах предлагается наземно-бортовой - спутниковый метод предусматривающий проведение следующих измерений:

- измерения влажного $\mathrm{CH}_{4}$ в нижней тропосфере, в приземном слое с помощью беспилотного летательного аппарата (БПЛА), снабженного измерительным прибором типа «Picarro CRDS»;

- спутниковые измерения GOSAT содержания влажного $\mathrm{CH}_{4}$ в нижней тропосфере;

- наземные измерения оптической толщины аэрозоля и влажности в нижней тропосфере (используя лидар).

Вкратце рассмотрим особенности вышеуказанных измерений.

Прежде всего, изложим физические основы предлагаемого метода. Согласно [3], существует достаточно сильная корреляция между объемом всего сжигаемого углеводородного газа и объемом элиттируемого $\mathrm{CO}_{2}$ и 
$\mathrm{CH}_{4}$ в процессе сжигания. Соответствующие линии регрессии показаны на рисунке 2.
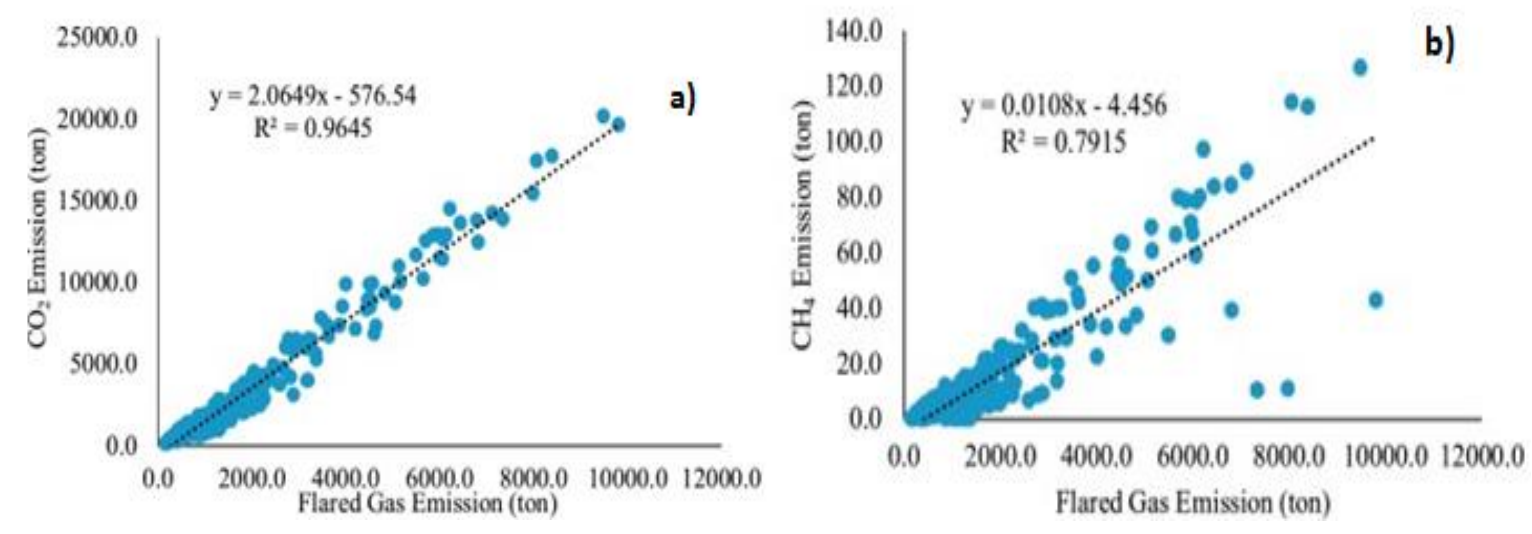

Рисунок 2. Регрессионные линии зависимостей между общим объемом сжигаемого газа и объемом эмитируемого $\mathrm{CO}_{2}$ (a) и $\mathrm{CH}_{4}$ (b) [3]

Схематическое представление предлагаемого метода наземно-бортовых - космических измерений показано на рисунке 3.

Как отмечается в работе [5], показания спектрального измерителя «Picarro CRDS» при измерении эмитируемого количества влажных $\mathrm{CO}_{2}$ и $\mathrm{CH}_{4}$ определяется следующим образом:

$$
\begin{aligned}
& \frac{C O_{2 w e t}}{C O_{2 d r y}}=1+a H+b H^{2} ; \\
& \frac{C H_{2 w e t}}{C H_{2 d r y}}=1+c H+d H^{2},
\end{aligned}
$$

где $a, b, c, d$ - постоянные отрицательные коэффициенты;

$H$ - молярная фракция водяных паров. 


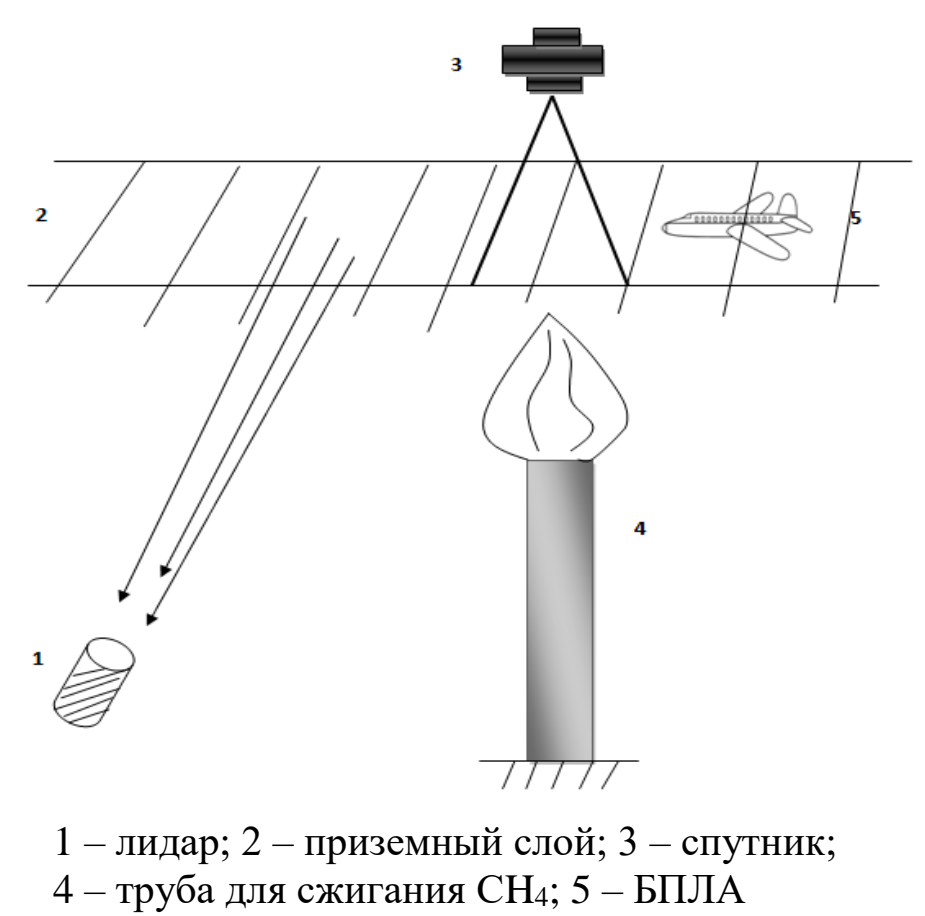

Рисунок 3. Схематическое представление предлагаемого наземно-бортового - космического метода определения парниковых эмиссионных показателей и общего сухого объема сжигаемого метана

Что касается зависимости измеряемой величины $\triangle X_{C H}(\%)$ от оптической толщины атмосферного аэрозоля, то согласно [1] имеется некоторая корреляционная связь между $\triangle X C H_{4}$ и $\tau_{\text {aer }}-$ оптической толщиной аэрозоля (рисунок 4).

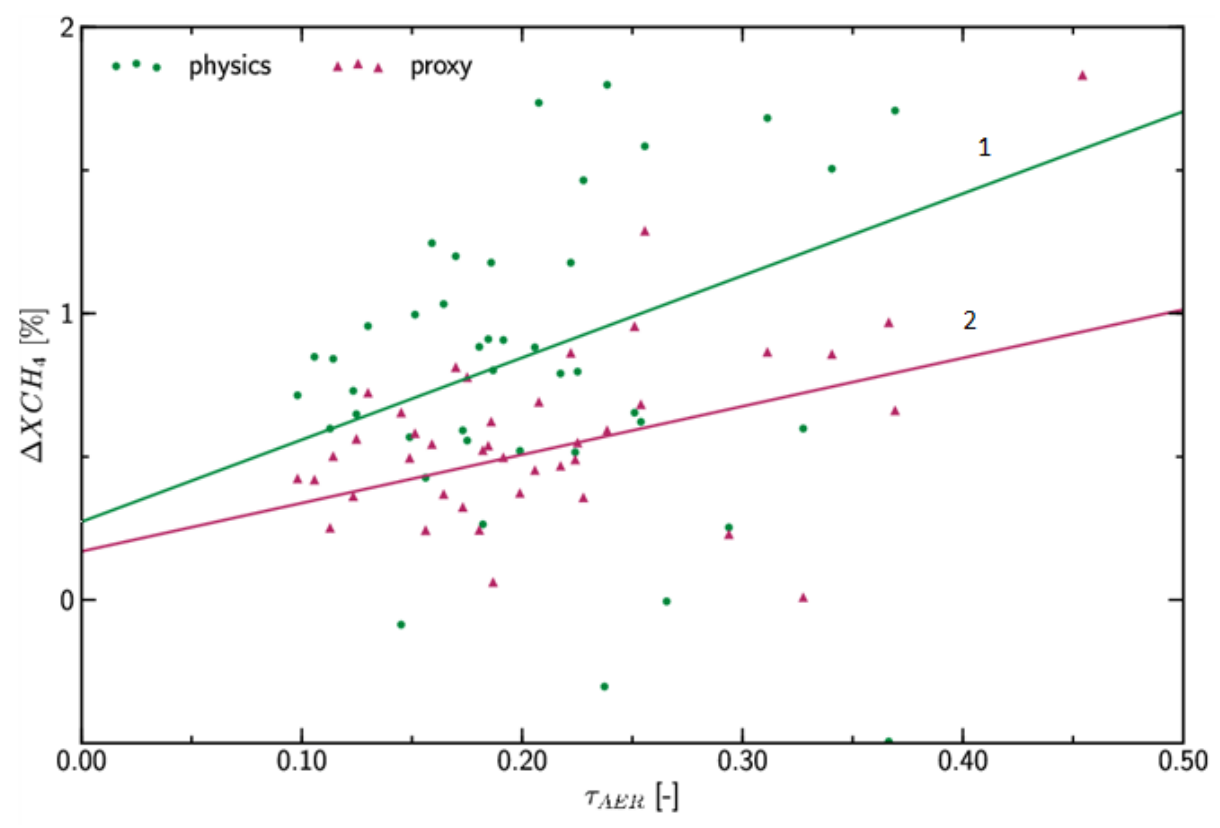

1 - для метода физических измерений; 2 - для прокси метода

Рисунок 4. Корреляционная связь между величиной $\triangle X C_{4}$ и оптической толщиной аэрозоля в приземной зоне 
Основным теоретическим положением, лежащем в основе предлагаемого наземно-бортового - спутникового метода является предположение о том, что основной причиной появления погрешности измерений, определяемой в качестве абсолютной разности показаний сенсоров, установленных на спутнике и БПЛА, является реально увлажненный атмосферный аэрозоль.

Предлагаемый на этой основе алгоритм разработанного наземно-бортового - космического метода может быть изложен в виде последовательности следующих шагов:

1. проводятся наземные лидарные измерения влажности и аэрозоля в исследуемом слое атмосферы;

2. по графикам, представленным на рисунке 4 определяется величина $\triangle X C H_{4}$ для прокси метода (кривая 2);

3. определяется величина $\mathrm{CH}_{4 w e t}$ для условия влажной атмосферы с помощью БПЛА;

4. величина $\triangle X_{C} H_{4}$ вычисляется по результатам лидарных аэрозольных измерений с использованием кривых, представленных на рисунке 4;

5. эта величина представляется в следующем виде:

$$
\Delta \mathrm{XCH}_{4}=\left|\mathrm{X}_{\mathrm{CH}_{4}}-\mathrm{CH}_{4 w e t}\right| \text {. }
$$

С учетом (1) и (4) получаем

$$
\Delta \mathrm{XCH}_{4}=\left|\left(\frac{\Omega_{\mathrm{CH}_{4 w e t}}}{\Omega_{\mathrm{CO}_{2 \text { wet }}}}\right) \cdot X_{\mathrm{CO} 2}-\mathrm{CH}_{4 \text { wet }}\right| .
$$

С учетом (3) и (5) получаем

$$
\Delta \mathrm{XCH}_{4}=\left|\left(\frac{\Omega_{\mathrm{CH}_{4 w e t}}}{\Omega_{\mathrm{CO}_{2 \text { wet }}}}\right) \cdot X_{C O 2}-\mathrm{CH}_{4 d r y}\left(1+\mathrm{CH}+d H^{2}\right)\right| .
$$

Из выражения (5) по результатам измерений $\Omega_{\mathrm{CH} 4 \mathrm{wet}}, \Omega_{\mathrm{CO} 2 \mathrm{dry}}$, Н и значения XCO2 можно вычислить величину $\mathrm{CH}_{4 \mathrm{dry}}$. 


\section{Формирование условия достоверности измерений}

Согласно графику, представленному на рисунке 2, a,

$$
\Omega_{C O_{2 w e t}}=k_{1} \cdot F_{1}
$$

где $k_{1}=$ const;

$F_{1}$ - объем сжигаемого газа также согласно графику, представленному на рисунке $2, b$,

$$
\Omega_{\mathrm{CH}_{4 \text { wet }}}=k_{2} \cdot F_{2},
$$

где $k_{1}=$ const;

$F_{1}$ - объем сжигаемого газа.

Примем в (5) $F_{1}=F_{2}=F_{0}$. Из (7), (8) получим

$$
\frac{\Omega_{\mathrm{CO}_{2 w e t}}}{k_{1}}=\frac{\Omega_{\mathrm{CH}_{4 w e t}}}{k_{2}} .
$$

С учетом (6) и (9) получим

$$
\Delta \mathrm{XCH}_{4}=\left|\frac{k_{2}}{k_{1}} \cdot X_{\mathrm{CO} 2}-\mathrm{CH}_{4 d r y}\left(1+C H+d H^{2}\right)\right| .
$$

Таким образом, выражение (10) позволяет проверить величину $\mathrm{CH}_{4 d r y}$, определяемую с помощью (6).

При выполнении условия (10) величину $F_{0}$ можно вычислить по формулам:

$$
\begin{aligned}
& F_{0}=\frac{\Omega_{\mathrm{CH}_{4}}}{k_{2}} ; \\
& F_{0}=\frac{\Omega_{\mathrm{CO}_{2}}}{k_{1}},
\end{aligned}
$$

где $X_{C H 4}-$ измеряется спутником; 
$\mathrm{CH}_{4}$ - измеряется БПЛА;

$X_{\mathrm{CO} 2}$ - модельная величина;

$\Omega_{\mathrm{CO} 2}$ - спутниковое измерение.

\section{Выводы}

Предложен наземно-бортовой - космический метод для сухого объема сжигаемого в факелах метана, а также для определения эмиссионных компонентов факелов углеводородного природного газа. Получена формула, позволяющая определить верность спутниковых, бортовых (БПЛА) и модельных измерений.

При подтверждении верности результатов измерений появляется возможность определить общий объем сжигаемого газа, составлен алгоритм реализации предложенного метода, включающего известный метод прокси $\mathrm{CH}_{4}$ измерений.

\section{Список используемых источников}

1. Emam E.A. Gas Flaring in Industry: an Overview // Petroleum and Coal. 2015. Vol. 57 (5). P. 532-555.

2. Fisher D., Wooster M.J. Multi-Decade Global Gas Flaring Change Inventoried Using the ATSR-1, ATSR-2, AATSR and ALSTR Data Records // Remote Sensing of Environment. 2019. Vol. 232. P. 111-298. DOI: 10.1016/j.rse.2019.111298.

3. Ziyarati M.T., Bahramifar N., Baghmisheh G., Younesi H. Greenhouse Gas Emission Estimation of Flaring in a Gas Processing Plant: Technique Development // Process Safety and Environmental Protection. 2019. Vol. 123. P. 289-298. DOI: 10.1016/j.psep.2019.01.008. 
4. Feng L., Palmer P.I., Bosch H., Parker R.J., Webb A.J., Corerreia C.S.C., Deutscher N.M., Domingues L.G., Feist D.G., Gatti L.V., Gloor E., Hase F., Kivi R., Liu Y., Miller J.B., Marino I., Suusmann R., Strong K., Uchino O., Wang J., Zahn A. Consistent Regional Fluxes of $\mathrm{CH} 4$ and $\mathrm{CO} 2$ Inferred from GOSAT Proxy XCH4: XCO2 Retrievals, 2010-2014 // Atmospheric Chemistry and Physics. 2017. Vol. 17. P. 4781-4797. DOI: 10.5194/acp-17-4781-2017.

5. Jacob D.J., Turner A.J., Maasakkers J.D., Sheng J., Sun K., Liu X., Chamce K., Aben I., McKever J., Frankenberg C. Satellite Observations of Atmospheric Methane and their Value for Quantifying Methane Emissions // Atmospheric Chemistry and Physics. 2016. Vol. 16. P. 14371-14396. DOI: 10.5194/acp-16-14371-2016.

6. Schepers D., Guerlet S., Butz A., Landgrave J., Frankenberg C., Hasekamp O., Blavier J-F., Deutscher N.M., Griffifth D.W.T., Hase F., Kyro E., Marino I., Sherlock V., Sussmann R., Aben I. Methane Retrievals from Greenhouse Gases Observing Satellite (GOSAT) Shortwave Infrared Measurements: Performance Comparison of Proxy and Physic Retrieval Algorithms // Journal of Geophysical Research. 2012. Vol. 117. Issue 10. P. D10307-1-D10307-14. DOI: 10.1029/2012JD017549.

7. Verhulst K.R., Karion A., Kim J., Saameh P.K., Keeling R.F., Newman S., Miller J., Sloop C., Pongetti T., Rao P., Wong C., Hopkins F.M., Yadav V., Weiss R.F., Duren R.M., Miller C.E. Carbon Dioxide and Methane Measurements from the Los Angeles Megacity Carbon Project - Part 1: Calibration, Urban Enhancements and Uncertainty Estimates // Atmospheric Chemistry and Physics. 2017. Vol. 17. P. 8313-8341. DOI: 10.5194/acp-17-8313-2017.

\section{References}

1. Emam E.A. Gas Flaring in Industry: an Overview. Petroleum and Coal, 2015, Vol. 57 (5), pp. 532-555. 
2. Fisher D., Wooster M.J. Multi-Decade Global Gas Flaring Change Inventoried Using the ATSR-1, ATSR-2, AATSR and ALSTR Data Records. Remote Sensing of Environment, 2019, Vol. 232, pp. 111-298. DOI: 10.1016/j.rse.2019.111298.

3. Ziyarati M.T., Bahramifar N., Baghmisheh G., Younesi H. Greenhouse Gas Emission Estimation of Flaring in a Gas Processing Plant: Technique Development. Process Safety and Environmental Protection, 2019, Vol. 123,pp. 289-298. DOI: 10.1016/j.psep.2019.01.008.

4. Feng L., Palmer P.I., Bosch H., Parker R.J., Webb A.J., Corerreia C.S.C., Deutscher N.M., Domingues L.G., Feist D.G., Gatti L.V., Gloor E., Hase F., Kivi R., Liu Y., Miller J.B., Marino I., Suusmann R., Strong K., Uchino O., Wang J., Zahn A. Consistent Regional Fluxes of $\mathrm{CH} 4$ and $\mathrm{CO} 2$ Inferred from GOSAT Proxy XCH4: XCO2 Retrievals, 2010-2014. Atmospheric Chemistry and Physics, 2017, Vol. 17, pp. 4781-4797. DOI: 10.5194/acp-17-4781-2017.

5. Jacob D.J., Turner A.J., Maasakkers J.D., Sheng J., Sun K., Liu X., Chamce K., Aben I., McKever J., Frankenberg C. Satellite Observations of Atmospheric Methane and their Value for Quantifying Methane Emissions. Atmospheric Chemistry and Physics, 2016, Vol. 16, pp. 14371-14396. DOI: 10.5194/acp-16-14371-2016.

6. Schepers D., Guerlet S., Butz A., Landgrave J., Frankenberg C., Hasekamp O., Blavier J-F., Deutscher N.M., Griffifth D.W.T., Hase F., Kyro E., Marino I., Sherlock V., Sussmann R., Aben I. Methane Retrievals from Greenhouse Gases Observing Satellite (GOSAT) Shortwave Infrared Measurements: Performance Comparison of Proxy and Physic Retrieval Algorithms. Journal of Geophysical Research, 2012, Vol. 117, Issue 10, pp. D10307-1-D10307-14. DOI: 10.1029/2012JD017549. 
7. Verhulst K.R., Karion A., Kim J., Saameh P.K., Keeling R.F., Newman S., Miller J., Sloop C., Pongetti T., Rao P., Wong C., Hopkins F.M., Yadav V., Weiss R.F., Duren R.M., Miller C.E. Carbon Dioxide and Methane Measurements from the Los Angeles Megacity Carbon Project - Part 1: Calibration, Urban Enhancements and Uncertainty Estimates. Atmospheric Chemistry and Physics, 2017, Vol. 17, pp. 8313-8341. DOI: 10.5194/acp-17-8313-2017.

\section{Сведения об авторах}

\section{About the Authors}

Асадов Ильгар Хикмет оглы, аспирант Национального аэрокосмического агентства, специалист местной структуры нефтяной компании CAMERON, г. Баку, Азербайджанская Республика

Ilgar H. Asadov, Post-graduate Student of National Aerospace Agency, Specialist of Local Structure of CAMERON COMPANY, Baku, Azerbaijan Republic e-mail: asadzade@rambler.ru

Рамазанов Вагиф Меджид оглы, доктор философии по экономике, доцент Азербайджанского государственного аграрного университета, г. Баку, Азербайджанская Республика

Vagif M. Ramazanov, Doctor of Philosophy in Economics, Associate Professor of Azerbaijan State Agrarian University, Baku, Azerbaijan Republic 